\title{
Unpolarised Transverse Momentum Dependent Distribution and Fragmentation Functions from recent HERMES and COMPASS SIDIS Multiplicities
}

\author{
M. Anselmino, ${ }^{a b}$ M. Boglione, ${ }^{a b}$ J.O. Gonzalez $\mathbf{H}^{* b}$, S. Melis, ${ }^{a}$ A. Prokudin. \\ ${ }^{a}$ Dipartimento di Fisica Teorica, Università di Torino, \\ Via P. Giuria 1, I-10125 Torino, Italy \\ ${ }^{b}$ INFN, Sezione di Torino, \\ Via P. Giuria 1, I-10125 Torino, Italy \\ ' Jefferson Laboratory, \\ 12000 Jefferson Avenue, Newport News, VA 23606, USA \\ E-mail: anselminodto.infn.it, boglionedto.infn.it, \\ joseosvaldo.gonzalez@to.infn.it, meliseto.infn.it, \\ prokudinejlab.gov
}

\begin{abstract}
The unpolarised transverse momentum dependent distribution and fragmentation functions (TMDs) are extracted from HERMES and COMPASS experimental measurements of semiinclusive deep inelastic scattering multiplicities for charged hadron production. A simple factorised functional form of the TMDs is adopted, with a Gaussian dependence on the intrinsic transverse momentum, which turns out to be quite adequate in shape.
\end{abstract}

XXII. International Workshop on Deep-Inelastic Scattering and Related Subjects Warsaw, Poland

28 April - 2 May 2014

\footnotetext{
* Speaker.
} 


\section{Contents}

1. Introduction and Formalism. 2

$\begin{array}{lll}\text { 2. } & \text { Results. } & 3\end{array}$

\section{Introduction and Formalism.}

We analyse the latest HERMES [1] and COMPASS [2] data on unpolarised semi-inclusive deep inelastic scattering (SIDIS) multiplicities, aiming at improving our knowledge of the unpolarised transverse momentum dependent distribution (TMD-PDF) and Fragmentation Functions (TMD-FF). We reconsider, with the support of the new, high statistics data, the first extraction of Ref. [3]; somewhat surprisingly, it turns out that the simple factorised form of the TMDs with the original, flavour independent, Gaussian parameterisation, still works rather well. However, the observed (Gaussian) dependence of the SIDIS cross section on the hadron transverse momentum, $P_{T}$, is generated by a combination of the (Gaussian) dependences in the quark TMD-PDF and TMDFF; thus, it is rather difficult to fix separately the parameters of the two Gaussians by studying only unpolarised multiplicities.

The unpolarised $\ell+p \rightarrow \ell^{\prime} h X$, SIDIS cross section in the TMD factorisation scheme, at order $\left(k_{\perp} / Q\right)$ and $\alpha_{s}^{0}$, in the kinematical region where $P_{T} \simeq k_{\perp} \ll Q$, reads $[4,5]$ :

$$
\begin{aligned}
& \frac{d \sigma^{\ell+p \rightarrow \ell^{\prime} h X}}{d x_{B} d Q^{2} d z_{h} d P_{T}^{2}}=\frac{2 \pi^{2} \alpha^{2}}{\left(x_{B} s\right)^{2}} \frac{\left[1+(1-y)^{2}\right]}{y^{2}} F_{U U}, \\
& F_{U U} \equiv \sum_{q} e_{q}^{2} \int d^{2} \boldsymbol{k}_{\perp} f_{q / p}\left(x, k_{\perp}\right) D_{h / q}\left(z, p_{\perp}\right) .
\end{aligned}
$$

In the $\gamma^{*}-p$ c.m. frame the measured transverse momentum, $\boldsymbol{P}_{T}$, of the final hadron is given at order $k_{\perp} / Q$ by $\boldsymbol{P}_{T}=z_{h} \boldsymbol{k}_{\perp}+\boldsymbol{p}_{\perp}$. The exact relations can be found in Ref. [3]. Furthermore, we assume for the $k_{\perp}$ and $p_{\perp}$ dependences a Gaussian form, factorized from other kinematical variables,

$$
f_{q / p}\left(x, k_{\perp}\right)=f_{q / p}(x) \frac{e^{-k_{\perp}^{2} /\left\langle k_{\perp}^{2}\right\rangle}}{\pi\left\langle k_{\perp}^{2}\right\rangle}, \quad D_{h / q}\left(z, p_{\perp}\right)=D_{h / q}(z) \frac{e^{-p_{\perp}^{2} /\left\langle p_{\perp}^{2}\right\rangle}}{\pi\left\langle p_{\perp}^{2}\right\rangle} .
$$

The integrated parton distribution functions (PDFs) and fragmentation functions (FFs), $f_{q / p}(x)$ and $D_{h / q}(z)$, can be taken from the available fits of the world data: in this analysis we used the CTEQ6L set for the PDFs [6] and the DSS set for the fragmentation functions [7]. In the simple 
Gaussian parameterisation, supported by a number of experimental evidences [8] as well as by dedicated lattice simulations [9], by inserting Eqs. (1.3) into Eq. (1.2), one obtains

$$
F_{U U}=\sum_{q} e_{q}^{2} f_{q / p}\left(x_{B}\right) D_{h / q}\left(z_{h}\right) \frac{e^{-P_{T}^{2} /\left\langle P_{T}^{2}\right\rangle}}{\pi\left\langle P_{T}^{2}\right\rangle}, \text { where }\left\langle P_{T}^{2}\right\rangle=\left\langle p_{\perp}^{2}\right\rangle+z_{h}^{2}\left\langle k_{\perp}^{2}\right\rangle .
$$

For the multiplicities, defined as $M_{n}^{h} \equiv \sigma^{S I D I S} / \sigma^{D I S}$ (see reference [10] for further details ), one gets

$$
\frac{1}{2 P_{T}} M_{n}^{h}\left(x_{B}, Q^{2}, z_{h}, P_{T}\right)=\frac{\pi \sum_{q} e_{q}^{2} f_{q / p}\left(x_{B}\right) D_{h / q}\left(z_{h}\right)}{\sum_{q} e_{q}^{2} f_{q / p}\left(x_{B}\right)} \frac{e^{-P_{T}^{2} /\left\langle P_{T}^{2}\right\rangle}}{\pi\left\langle P_{T}^{2}\right\rangle},
$$

with $\left\langle P_{T}^{2}\right\rangle$ given in Eq. (1.4). Notice that $\left\langle k_{\perp}^{2}\right\rangle$ and $\left\langle p_{\perp}^{2}\right\rangle$ are the free parameters of our fit.

\section{Results.}

We first consider the HERMES multiplicities of [1]. To make sure we work in the region of validity of our simple version of TMD factorization, Eq. (1.5), we restrict the kinematical range of the analysis to the region $z<0.6, Q^{2}>1.69 \mathrm{GeV}^{2}, 0.2<P_{T}<0.9 \mathrm{GeV}$.

Moreover, in our fit we do not include the kaon production data points, since the precision and accuracy of the kaon data sample do not further constrain the values of the free parameters (See Section 3.1 of [10] for further details in the selection of the data).

The details of the fits are presented in Table 1. Fig. 1 shows the comparison between the data and the model, for proton target and positive pions. Similar results are obtained for other negative pions and for deuteron target [10]. It is also worth noticing that we do not use any overall normalisation constant as an extra free parameter.

embeds the crucial features of the data, both in

A careful look at the plot in Fig. 1 shows that the description of the HERMES measurements is indeed satisfactory: the Gaussian parameterisation embeds the crucial features of the data, both in shape and size, over a broad kinematical range. The resulting value of $\chi_{\mathrm{dof}}^{2}$, still quite sizeable, is somehow expected, given the uncertainties on the collinear fragmentation functions.

\section{HERMES}

\begin{tabular}{cccccc}
\hline Cuts & $\chi_{\text {dof }}^{2}$ & n. points & {$\left[\chi_{\text {point }}^{2}\right]^{+^{+}}$} & {$\left[\chi_{\text {point }}^{2}\right]^{\pi^{-}}$} & Parameters \\
\hline $\begin{array}{c}Q^{2}>1.69 \mathrm{GeV}^{2} \\
0.2<P_{T}<0.9 \mathrm{GeV} \\
z<0.6\end{array}$ & 1.69 & 497 & 1.93 & 1.45 & $\left\langle k_{\perp}^{2}\right\rangle=0.12 \pm 0.01 \mathrm{GeV}^{2}$ \\
& & & & & \\
\hline
\end{tabular}

Table 1: $\chi^{2}$ values of our best fits, following Eqs. (1.4) and (1.5), of the experimental HERMES measurements of the SIDIS multiplicities $M_{n}^{h}\left(x_{B}, Q^{2}, z_{h}, P_{T}\right)$ for $\pi^{+}$and $\pi^{-}$production, off proton and deuteron targets. We show the total $\chi_{\text {dof }}^{2}$ and, separately, the $\chi_{\text {point }}^{2}$ for $\pi^{+}$and $\pi^{-}$data. CTEQ6 PDFs and DSS FFs are used. Notice that the errors quoted for the parameters are statistical errors only, and correspond to a 5\% variation over the total minimum $\chi^{2}$. 


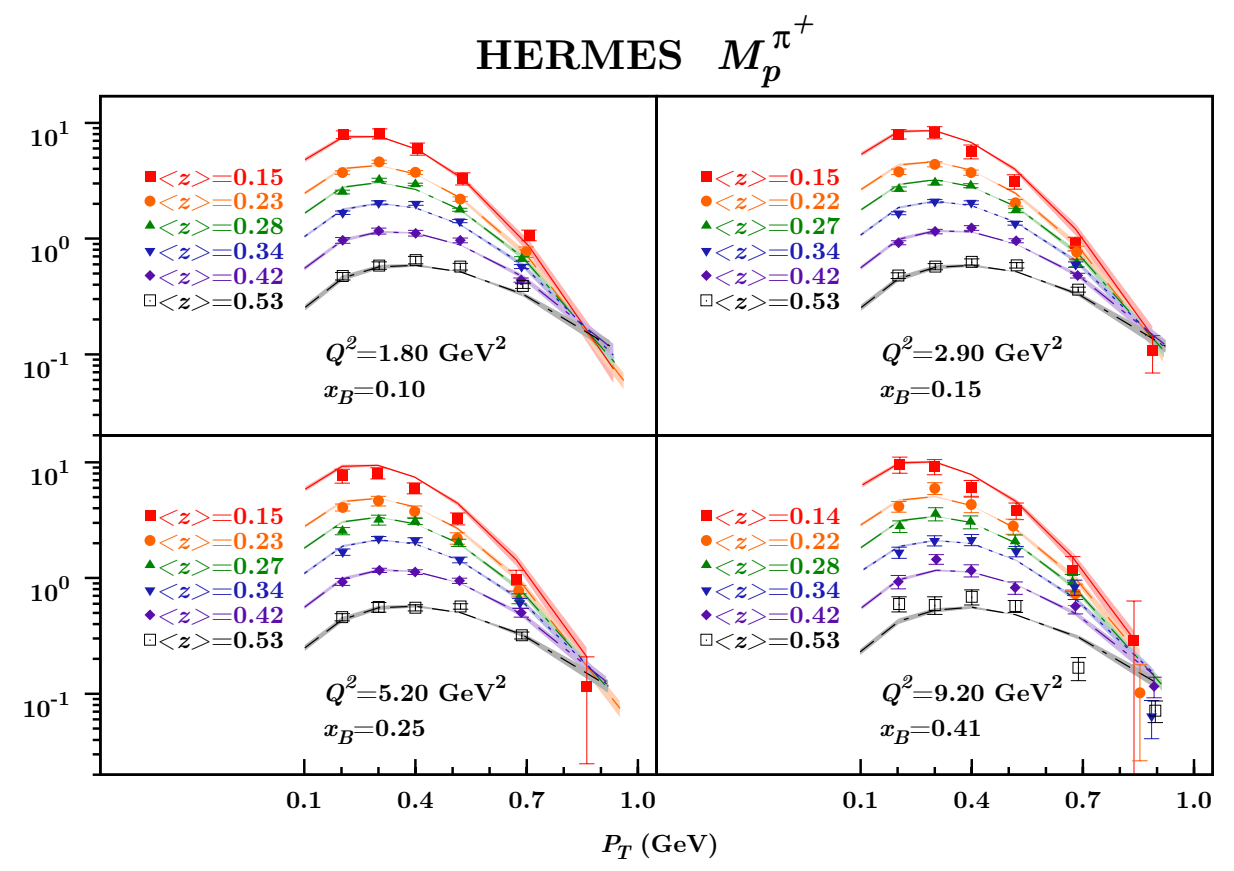

Figure 1: The multiplicities $M_{p}^{\pi^{+}}$obtained from Eqs.(1.4) and (1.5), with the parameters of Table (1), are compared with HERMES measurements for $\pi^{+}$SIDIS production off a proton target [1]. The shaded uncertainty bands correspond to a 5\% variation of the total $\chi^{2}$.

We now consider the COMPASS SIDIS multiplicities of Ref. [2] and best fit them separately. We apply the same cuts as those used for the HERMES set of data, (see Tables 1 and 2), with the same motivations, which reduce the number of fitted data points to 5385 . Our resulting $\chi_{\text {dof }}^{2}$ value is presented in Table 2 and turns out to be much larger $\left(\chi_{\text {dof }}^{2}=8.54\right)$ than that obtained by fitting the HERMES multiplicities.

By carefully inspecting the $\chi^{2}$ contributions of each individual bin, we realised that major improvements of our description of COMPASS data cannot actually be achieved by modifying the Gaussian widths, nor by making it more flexible. Our simple Gaussian model can actually reproduce the shape of the data, even over a large kinematical range; rather, the difficulties of the fit seem to reside in the normalisation. In fact, the data exhibits an approximately linear $y$ dependence in the normalisation. To include this effect, we introduced an overall factor of the form $N_{y}=A+B y$, where $A$ and $B$, are two new parameters assumed to be universal and flavour independent.

With this parameterisation the quality of our best fit improves very significantly, resulting in a total $\chi_{\text {dof }}^{2}$ of 3.42 , corresponding to $A=1.06 \pm 0.06$ and $B=-0.43 \pm 0.14$ and only very slightly different values of the Gaussian widths with respect to those previously obtained, as seen in Table 2.

The results of our best fit including this normalisation factor, for positively charged hadronic production, are presented in Fig. 2. Similar results are obtained for negative hadrons [10]. Notice that this normalisation issue is not observed in the HERMES multiplicities and its origin, at present, cannot easily be explained; it might be related to experimental acceptance effects and deserves 
COMPASS

\begin{tabular}{|c|c|c|c|c|c|}
\hline Cuts & $\chi_{\mathrm{dof}}^{2}$ & n. points & {$\left[\chi_{\text {point }}^{2}\right]^{h^{+}}$} & {$\left[\chi_{\text {point }}^{2}\right]^{h^{-}}$} & Parameters \\
\hline $\begin{array}{c}Q^{2}>1.69 \mathrm{GeV}^{2} \\
0.2<P_{T}<0.9 \mathrm{GeV} \\
z<0.6\end{array}$ & 8.54 & 5385 & 8.94 & 8.15 & $\begin{array}{l}\left\langle k_{\perp}^{2}\right\rangle=0.61 \pm 0.20 \mathrm{GeV}^{2} \\
\left\langle p_{\perp}^{2}\right\rangle=0.19 \pm 0.02 \mathrm{GeV}^{2}\end{array}$ \\
\hline $\begin{array}{c}Q^{2}>1.69 \mathrm{GeV}^{2} \\
0.2<P_{T}<0.9 \mathrm{GeV} \\
z<0.6 \\
N_{y}=A+B y\end{array}$ & 3.42 & 5385 & 3.25 & 3.60 & $\begin{array}{c}\left\langle k_{\perp}^{2}\right\rangle=0.60 \pm 0.14 \mathrm{GeV}^{2} \\
\left\langle p_{\perp}^{2}\right\rangle=0.20 \pm 0.02 \mathrm{GeV}^{2} \\
A=1.06 \pm 0.06 \\
B=-0.43 \pm 0.14\end{array}$ \\
\hline
\end{tabular}

Table 2: $\chi^{2}$ values of our best fits, following Eqs. (1.4) and (1.5), of the experimental COMPASS measurements of the SIDIS multiplicities $M_{n}^{h}\left(x_{B}, Q^{2}, z_{h}, P_{T}\right)$ for $h^{+}$and $h^{-}$production, off a deuteron target. We show the total $\chi_{\mathrm{dof}}^{2}$ and, separately, the $\chi_{\mathrm{dof}}^{2}$ for $h^{+}$and $h^{-}$data. CTEQ6 PDFs and DSS FFs are used. Notice that the errors quoted for the parameters are statistical errors only, and correspond to a $5 \%$ variation over the total minimum $\chi^{2}$.

further studies. It is worth noticing that the persistent large value of $\chi^{2}$, even after correcting with the factor $N_{y}$, is mainly due the panels, in Fig. 2, corresponding to the lowest values of $y$.

Our simple Gaussian parameterisation delivers a satisfactory description of the HERMES data points over large ranges of $x, z, P_{T}$ and $Q^{2}$, selected according to Table 1. These measurements are well described by a TMD Gaussian model with constant and flavour independent widths, $\left\langle k_{\perp}^{2}\right\rangle$ and $\left\langle p_{\perp}^{2}\right\rangle$, which we extract as (the only two) free parameters of our fit.

By fitting COMPASS data, the Gaussian shape of the $P_{T}$ dependence is qualitatively well reproduced. Furthermore, we found that this data set is consistent with an overall normalisation factor linear in $y$, which would be very difficult to accommodate in a QCD driven scheme, even considering scale evolution [10]. This effect needs further investigation, both on the theoretical and experimental sides. In both data sets, we considered flavour dependence as well as $x$-dependences for the TMD distribution and $z$-dependence for the TMD fragmentation function. We found no significant improvement in the fit. Additionally, attempts to include scale-dependence produced no evidence for scale evolution, for the HERMES data, and a marginal improvement for the COMPASS data, which cannot outperform the considered normalisation factor $N_{y}=A+B y$. For details on these last points, we refer the reader to [10]. (AP's work is supported by the U.S. Department of Energy under contract No. DE-AC05-06OR23177).

\section{References}

[1] A. Airapetian et al. (HERMES Collaboration), Phys. Rev. D87 (074029) 2013 [arxiv: 1212 . 54 07 ]

[2] C. Adolph et al. (COMPASS), Eur. Phys. J. C73 (2531) 2013 [arxiv: 1305 . 7317]

[3] M. Anselmino, M. Boglione, U. D’Alesio, A. Kotzinian, F. Murgia, A. Prokudin, Phys. Lett. D71 (074006) 2005 [hep-ph / 0501196 ] 


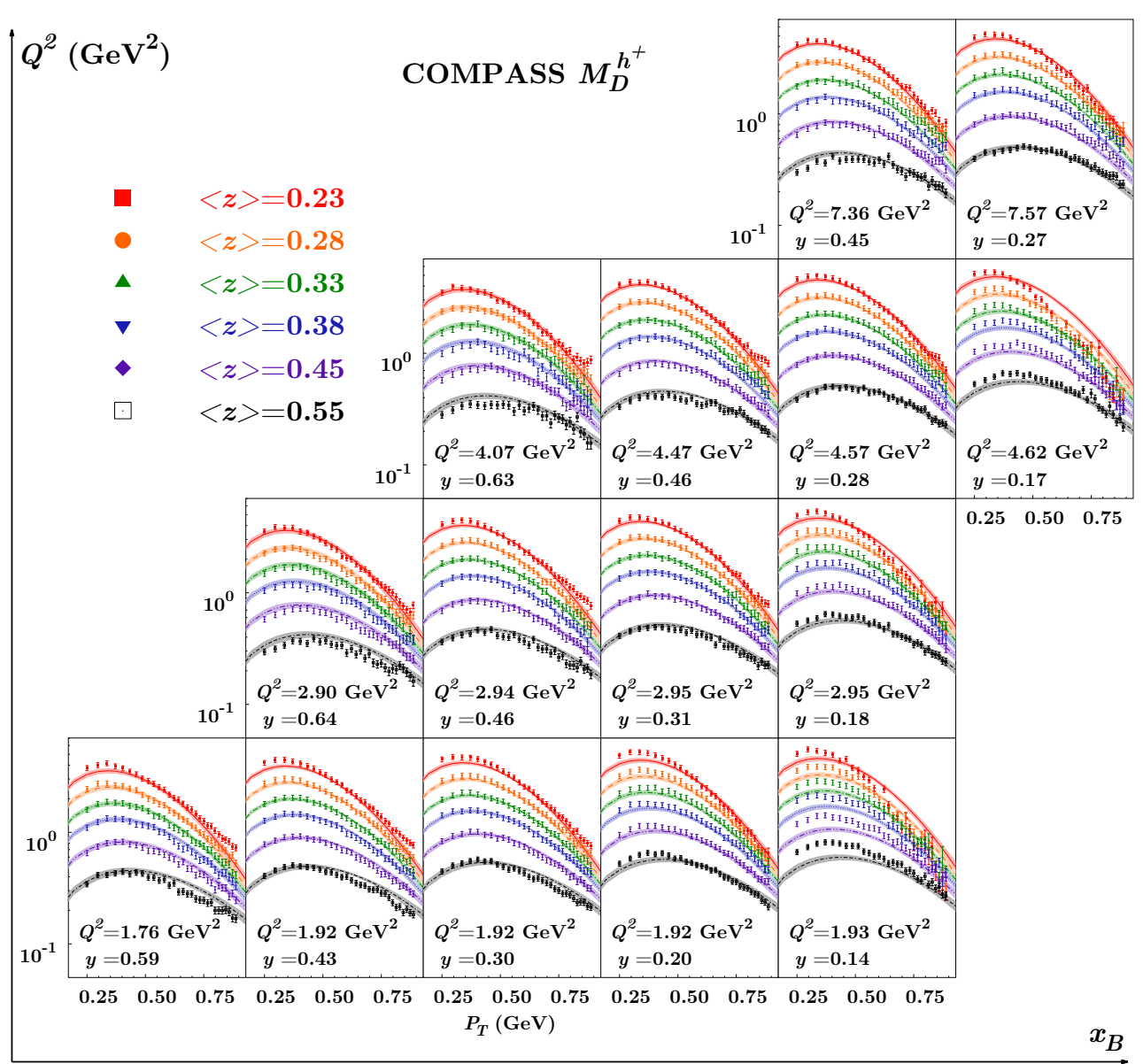

Figure 2: The multiplicities obtained from Eqs.(1.4) and (1.5), including the $y$-dependent normalisation factor of the second entry in Table 2, are compared with the COMPASS measurements for $h^{+}$SIDIS production off a deuteron target. The shaded uncertainty bands correspond to a $5 \%$ variation of the total $\chi^{2}$.

[4] A. Bacchetta, M. Diehl, K. Goeke, A. Metz, P. J. Mulders, et al., JHEP 0702 (093) 2007 [hep-ph/0611265]

[5] M. Anselmino, M. Boglione, U. D’ Alesio, S. Melis, F. Murgia, et al., Phys. Rev. D83 (114019) 2011 [arxiv:1101.1011]

[6] J. Pumplin, D. Stump, J. Huston, H. Lai, P. M. Nadolsky, et al., JHEP 0207 (012) 2002 [hep-ph/0201195]

[7] D.de Florian, R.Sassot, and M.Stratmann., Phys. Rev. D75 (114010) 2007 [hep-ph / 0703242 ]

[8] P Schweitzer, T Teckentrup, A Metz., Phys. Rev. D81 (094019) 2010 [arxiv: 1003.2190 ]

[9] B. U. Musch et al., PoS LAT2007 (155) 2007 [arxiv: 0710 . 4423]

[10] M. Anselmino, M. Boglione, J.O. Gonzalez H. , S. Melis, A. Prokudin, JHEP 1404 (005) 2014 [arxiv:0710.4423]

[11] D. Boer, M. Diehl, R. Milner, R. Venugopalan, W. Vogelsang, et al., 2011 [arXiv: 1108 . 1713]

[12] X.-d. Ji, J.-P. Ma and F. Yuan, Phys. Lett. B597 (299) 2004 [hep-ph/ 0405085 ] 